\title{
Versatile Diode
}

\author{
Prof. (Dr.) Utpal Kumar Das ${ }^{1}$ MDS, Dr. Niladri Maiti ${ }^{2}$ BDS \\ '(, Head of the Department, Department of Conservative Dentistry \& Endodontics, Guru Nanak Institute of \\ Dental Science and Research, Kolkata, India). \\ 2(, Post Graduate Student, Department of Conservative Dentistry \& Endodontics, Guru Nanak Institute of \\ Dental Science and Research, Kolkata, India).
}

\begin{abstract}
Among the various laser types with corresponding wavelengths, diode laser systems quickly began establishing themselves as compact, competitively priced and versatile additions to the dentist's repertoire. Research has shown that near infrared (NIR) wavelengths have high absorption in haemoglobin. This fact gives NIR laser the ability to precisely and efficiently cut, coagulate, ablate or vaporize the target tissue. The added advantage of performing desired procedures is the sealing of small blood and lymphatic vessels, resulting in hemostasis and reduced post-operative edema, disinfection of target tissue due to local heating and production of eschar layer and decreased amount of scarring due to decreased post-operative tissue shrinkage. Research has shown that Diode laser is one of the most versatile with regard to the number of possible treatment options and can be effectively used in the field of soft tissue surgery, periodontics, endodontics, tooth whitening etc. The versatility of the instrument, combined with the latest achievements in diode laser technology, compact design and affordability, should appeal to dental professionals seeking to optimize the procedures they currently perform.
\end{abstract}

Keywords: Diode Laser, Emisssion Modes, Optic Fiber, Target Tissue.

\section{Introduction}

There was a time when the academics in dentistry spurned a new technology that had been advancing for more than a decade. Individuals from the institutions criticised the cost, limited utility and lack of evidence (Dentistry 1991; Cobb 1997). This new technology is the use of lasers in dentistry. It is for good reason that the teaching institutions could not recommend this technology for general use due to initial problems and the availability of traditional, cheaper approaches to solving common dental problems. However, forward thinking universities have used laser technology as a comparative approach to traditional methods to see if their use can possibly enhance treatment outcomes.

Dental practitioners are being encouraged by manufacturers to invest in laser technology to enhance the commercial success of their practices as patient expectations increase (DeShields 1995; Weiner 2004) [1]

\section{History}

In 1917, Albert Einstein first theorized about the process which makes lasers possible called "Stimulated Emission."[2]

Theodore Maiman at the Hughes Research Laboratories had exposed an extracted tooth to his ruby laser in 1960, the breakthrough for lasers in the field of the dentistry came in the mid 1990s, with various laser types (Nd:YAG, Er,Cr:YSGG, Er:YAG, CO2) with corresponding wavelengths (1064 nm, 2780 nm, 2940 nm, 10600 $\mathrm{nm}$ ) becoming available to the dentists to address their needs for hard and soft tissue procedures. [3](Fig. no. $1,2$,

\section{Diode In Picture}

October 1962: Nick Holonyak Jr., a consulting scientist at a General Electric Co. lab in Syracuse, N.Y., publishes his work on the "visible red" GaAsP (gallium arsenide phosphide) laser diode, a compact, efficient source of visible coherent light that is the basis for today's red LEDs used in consumer products such as CDs, DVD players and cell phones .

1963: Herbert Kroemer of the University of California, Santa Barbara, and the team of Rudolf Kazarinov and Zhores Alferov of A.F. Ioffe Physico-Technical Institute in St. Petersburg, Russia, independently propose ideas to build semiconductor lasers from heterostructure devices. The work leads to Kroemer and Alferov winning the 2000 Nobel Prize in physics. [4]

\section{Why Diode??}

The attraction of diode lasers over all other types of laser is their extreme compactness, and ease of operation. The diodes themselves have sub-millimetre dimensions, and their high efficiency means that they 
have small power supplies. For example a $10 \mathrm{~mW}$ diode laser might require $100 \mathrm{~mW}$ of electrical power, easily provided by a battery, whereas a gas laser such as a He-Ne laser would require $10 \mathrm{~W}$, and thus must be mains powered. At the high power end the differences are even more stark. A $40 \mathrm{~W}$ copper vapour laser, one of the most efficient gas lasers, needs $5 \mathrm{~kW}$ of electrical power. This means using a three phase supply. By contrast a $40 \mathrm{~W}$ diode array might require $200 \mathrm{~W}$ of electrical power, less than an average computer supply. Thus the high efficiency not only reduces the energy costs of running the lasers, but also dramatically reduces the size, cost, and complexity of the associated power supplies. (Fig. no. 3, 4)

\section{Effects On Tissue}

Lasers have four different interactions with the target tissue. These interactions will depend on the optical properties of the tissue.

\section{v.i Photobiologic Interactions}

The first effect is reflection, which is simply a beam redirecting itself off the tissue surface, having no effect on the target tissue.

The second interaction is absorption of laser energy by the intended target tissue. This effect is desirable and the amount of energy that is absorbed by the tissue depends on the tissue characteristics, such as pigmentation and water content, and on the laser wavelength and emission mode.

The third interaction is transmission of the laser energy directly through the tissue with no effect on the target tissue.

The fourth interaction is scattering. Scattering of the reflected light weakens the intended energy and possibly produces no useful biological effect. [5]

\section{v.ii. Photochemical Interactions}

The basic principle of photochemical process is that specific wavelengths of laser light are absorbed by naturally occurring chromophores which are able to induce certain biochemical reactions. Photosensitive compounds when exposed to laser energy can produce a single oxygen radical for disinfection of endodontic canals. [6]

\section{v.iii. Photothermal Interactions}

The radiant energy absorbed by tissue substances are transformed into heat energy, which produce the tissue effect. [5]

\section{v.iv Photomechanical and photo electrical interactions}

These include photodisruption, photoplasmolysis and photoacoustic interactions. In photoacoustic effects, the pulse of laser energy on the dental tissues can produce a shock wave. When this shock wave explodes or pulverizes the tissue, it creates an abraded crater. Photoelectrical effect includes photo plasmolysis, which describes how the tissue is removed through formation of electrically charged ions. [5]

\section{Science Behind Diode Laser}

Only solid material active media (e.g. GaAlAs - Gallium Aluminum Arsenide) is used in diode lasers. Because of the crystalline nature of the active medium, the ends of the crystal can be selectively polished relative to internal refractive indices to produce totally and partially reflective surfaces thus serving the same function as the optical resonators of larger laser systems. The discharge of current across the active medium releases photons from the active medium, finally resulting in the generation of laser light of a specific wavelength, which is determined by the active medium used. (Fig. no.5)

At the present, each diode "chip" produces relatively low-energy output. Some low power diode lasers, operating in milliwatt range, are usually being advertised for low level laser therapy (LLLT). In order to achieve the power necessary for various dental procedures (e.g. soft tissue surgery), todays dental diode lasers employ banks of individual diode chips in parallel to achieve the appropriate power levels (several watts). Some dental diode lasers can also be set to lower power (milliwatt range) and can also perform LLLT procedures. [3]

\section{Emission Modes}

Lasers are said to be running in either continuous wave $(\mathrm{CW})$ or pulsed mode. This relates to the rate of emission of laser light with time and the prime benefit of a pulsed mode will be the capacity of the target tissue to cool between successive pulses. The CW mode is generally the fastest way to ablate tissues but heat can build up and cause collateral damage to the target and adjacent tissues. Modern dental diode lasers can operate in both $\mathrm{CW}$ and pulsed mode. The factors that determine the average power when the diode laser is operating in pulsed mode are the current power setting and the duty cycle setting. Duty cycle is a periodic 
phenomenon defined as the ratio of the duration of the phenomenon (pulse width) in a given period to the period (reciprocal value of the current frequency setting - number of pulses per second). [3]

\section{vii.i Delivery to the Target Tissue:}

Dental diode lasers employ a flexible optic fiber (usually inserted into an appropriate handpiece for comfortable handling) to deliver the treatment beam to the desired area. There are a number of things to consider when using an optic fiber. When using parameters mentioned in application notes or in research papers, always note the diameter of the fiber described in those papers. Using a smaller diameter fiber will increase the power density at the fiber tip. As a result, you may need to decrease the power setting. Increasing the power may be required when using a larger diameter fiber. As a rule of thumb, in order to achieve the same rate of work after changing fiber diameters, a smaller diameter fiber will require less power and conversely, a larger diameter will require more power.[3]

\section{Optic Fiber And Its Modifications}

Do not continue to use the fiber tip once you have observed that the tip has a blackened appearance that is greater in length than $2-4 \mathrm{~mm}$ from the previous cleave spot. The protein debris of gingival tissue accumulates on the tip during surgery and retains extreme heat that can cause rapid tip deterioration and subsequent breakage.

The fiber itself has three components:

- Jacket

- Cladding

- Quartz/silica fiber

Jacket the protective cover for the fiber system and usually is made of a synthetic material that is clear or white in color. Cladding is the material on the outside of the quartz/silica fiber that is used to block the lateral escape of laser energy as it traverses the fiber.

Quartz/silica fiber is fairly flexible but can be broken if bent into a small circle or bent at an angle of 90 degrees.

\section{viii.i Stripping the Fiber}

The fiber's jacket is removed using a "stripper. Begin by selecting enough jacket so that when it is removed, you will have approximately $3 / 8$ " of bare fiber exposed. Place the fiber in the stripper and grasp that portion of the fiber that will have the jacket removed between your thumb nail and index finger.

\section{viii.ii Cleaving the Fiber}

The cleave is made after stripping off the jacket to expose approximately $1 / 4 "-3 / 8$ " of bare fiber. The cleave should be made at a point approximately $1 / 2 "(8-10 \mathrm{~mm})$ from the site of the previous cleave so that there is no visible discoloration of the tip.

\section{Movement Of The Fiber Tip During Treatment}

Tissue charring is an undesirable side effect of too much power and/or the tip moving too slowly. Always use the least amount of power necessary to complete your procedure and move the fiber tip using short 1-2 mm "paint brush" type strokes and move quickly when working on soft tissue. Finally, regularly check the condition of the optical fiber. Always cleave the fiber tip after it becomes blackened (2-4 $\mathrm{mm}$ from the tip), because tissue debris accumulate on the tip during surgery and this causes the fiber tip to retain extreme heat and begins to act as a "branding iron". This can lead to unwanted tissue heating and can lead to rapid tip deterioration and subsequent breakage. Faster laser beam movement will also reduce heat build-up in the target tissue and aid thermal relaxation.

\section{x.i Soft-Tissue Surgery}

\section{Clinical Applications}

Include the removal of fibromata, labial and lingual frenectomies, small hemangiomata, mucocele, denture granulomata, and treatment of nonerosive lichen planus, aphthae and herpes lesions. $(7,8,9)$ With regard to diode laser surgery, the laser handpiece tip is generally held very close to the tissue surface. This allows the laser energy to effect the incision and minimizes the build-up of debris on the tip, which can lead to unwanted thermal damage to the tissue. For most minor intra-oral surgical procedures, the recommended average power setting is in the range of 2-4 $\mathrm{W}$ [8].

Diode laser transverses the epithelium and penetrates $2-6 \mathrm{~mm}$ into the tissue. When laser cutting is in progress, small blood and lymphatic vessels are sealed due to the generated heat, thereby reducing or eliminating bleeding and edema. Denatured proteins within tissue and plasma are the source of the layer termed 
"coagulum" or "char", which is formed because of laser action and serves to protect the wound from bacterial or frictional action. Clinically, during 48-72 hours post-surgery, this layer becomes hydrated from saliva, swells and eventually disintegrates to later reveal an early healing bed of new tissue.[8]

Additionally, gingival hyperplasia, tooth exposure and hyperpigmentation a range of gingival adaptation procedures, both to allow restorative procedures and to allow access to restorative margins during restorative procedures. [8] The laser energy will act primarily as a means of incision, excision or ablation, with the same advantages over the scalpel that were mentioned previously (no or minimal bleeding, no sutures, less chance for infection of the wound). When possible, any laser surgical procedure in and around the gingival cuff should seek to preserve a biological width (the zones of connective and epithelial tissues attached to the tooth), minimum $3 \mathrm{~mm}$ in depth, which will help to maintain gingival margin stability, alveolar bone height and health and prevent overgrowth.[1011,12]

Power settings of 1.5-3.0 watts with intervals should be optimal for most, if not all gingival procedures. [8] Again care must be taken to avoid thermal damage to the underlying periosteum and bone, together with root surface at gingival margin levels. Therefore assessment of the thickness, vascularity and position of any target gingival tissue, together with an assessment of adjacent bone and tooth tissue, is recommended. Also, to minimize the buildup of carbonized debris, post-ablation tissue should be discarded using a curette, damp cotton wool or gauze. [8]

\section{x.ii Periodontal Therapy with Diode Laser}

Optic fiber delivery systems, with 200-320 $\mu \mathrm{m}$ fiber diameters, enable extremely easy access into the periodontal pocket. After hard and soft deposits have been removed through scaling and/or root-planing, the pocket architecture is re-assessed, with emphasis on the depth. The fiber is then measured to a distance of one to two millimeters short of the pocket depth and is inserted at an angle to maintain contact with the soft tissue wall at all times. The fiber is then used in light contact, sweeping mode to cover the entire soft tissue lining. Power setting of $0.8-1 \mathrm{~W}$ should suffice to ablate the epithelial lining. Start with the ablation near the base of the pocket and slowly proceed upwards. Often some bleeding of the pocket site will occur, possibly due to damage to the inflamed pocket epithelium, but in terms of laser hemostasis, the power levels used are low and aimed at removing the epithelial surface and disinfecting the pocket .

The fiber tip should be regularly inspected and cleaned with a damp sterile gauze or cleaved in order to prevent the buildup of debris on the fiber tip. The treatment time per pocket should be around 20-30 s, amounting possibly to 1-2 minutes per tooth site. Retreatments should follow at weekly intervals during the maximum four week period. [13]

\section{x.iii Diode Laser in Implantology}

Dental diode laser can be used for second stage implant recovery and the treatment of peri-implantitis. In second stage implant recovery care must be exercised to avoid contact with the implant body. Soft tissue ablation leads to precise and predictable healing and the procedure can usually be performed with the use of a topical anesthetic. The appropriate power setting for the removal of gingival tissue overlying the implant cover screw is $1-2 \mathrm{~W}$. The advantages of using a diode laser to perform this procedure are easier visual access to the cover screw due to hemostasis and the production of the protective coagulum to aid in healing and patient comfort. [14]

Peri-implantitis is described as one of the most important causes of implant loss and is not restricted to any one type of implant design or construction $[15,16]$. It can be recognized as a rapidly progressive failure of osseo-integration [17] in which the production of bacterial toxins leads to inflammatory change and bone loss.[18]. Always, an assessment must be made to determine the causative factors associated with the condition (infection, implant overloading, occlusion and other local, systemic and life-style factors), to establish whether the implant can be saved. [14]

Curettage of granulation tissue is especially important. Research has shown that a diode laser can be used to perform the procedure with the added bonus of disinfecting the treated area. Use of appropriate coolant (eg. water spray) is needed to avoid any detrimental heat effects to the surrounding tissues. $[14,19]$ Effective power range is from $1-1.5 \mathrm{~W}[19]$.

\section{x.iv Diode Laser in Endodontics}

The bactericidal effect of conventional alternating rinsing in the root canal preparation with $\mathrm{H} 2 \mathrm{O} 2 / \mathrm{NaOCl}$ is proven (BYSTRÖM et al., 1985; ORSTAVIK et al., 1990; SHIH et al., 1970; SMITH et al, 1986; SPANGBERG et al., 1973). However, the extent of the micro -organism reducing effect varies from study to study. BYSTRÖM et al., 1983, were only able to observe an $80 \%$ bacterial reduction after five treatment sessions . While in addition, these effects can only be achieved with root canals of up to ISO 30, and not with curved roots.[20,21] 
The fine diameters of optic fibers (200-320 $\mu \mathrm{m})$ enable effective delivery of laser light to the root canal to help with reduction of bacterial contamination. After measuring the canal depth, the optic fiber should be inserted in the prepared root canal down to the apex - in no case further. The optic fiber is then led in slow, circular, spiral-forming movements from the apical to the coronal part, while the laser is activated. The procedure should be repeated four times for five seconds. Be cautious to always keep the fiber-optic beam delivery tip moving when the laser is activated to avoid excessive temperature rise on the tooth surface, which can be detrimental to the tissues surrounding the tooth. [22, 23]

\section{X.v Diode laser in pulp capping}

Tuner and Hode stated the use of diode laser in pulp capping and pulp amputation. They appear to stimulate odontoblast calcium and collagen production leading to secondary dentin formation. Diode laser in continuous mode set between $1.0 \mathrm{~W}$ upto $2.0 \mathrm{~W}$ can be used in pulp capping. Optical fiber of 320 micron in used.

\section{x.vi Teeth Whitening using the Dental Diode Laser:}

Teeth whitening procedures continue to grow in popularity due to the increased desire for whiter teeth with increasing number of articles being published on the subject in the popular press and on television in regular intervals. This has resulted in renewed interest from the dental profession in the process of teeth whitening, as the procedure itself is relatively simple and non- invasive to carry out. Current bleaching systems are based primarily on hydrogen peroxide (HP) or carbamide peroxide (CP). These bleaching systems usually exist in a form of a gel which is applied on the tooth surface and activated via light, for example. Activation of HP causes formation of free radical ions, which immediately seek available targets to react with. Long-chained molecules that "stain" the tooth react with the free radicals, altering the optical structure of the molecule and creating a different optical structure. The stain on the tooth surface disappears, or the large molecules become virtually dissociated into smaller, shorter chained molecules, giving the tooth surface a brighter appearance.

Laser light also generates heat on the tooth surface. In order to prevent excessive conduction of heat to the pulp and avoid pulpal necrosis, proper laser power must be used and according to the recently published research, an up to $2 \mathrm{~W}$ setting should be well within safety margins with regard to the pulp tissue as well as being high enough to accelerate the bleaching process by causing the breakdown of the HP gel to reactive free radicals that penetrate the tooth to cause the oxidation of stain molecules within the tooth structure. [24]

\section{Conclusion}

In today's dental diode laser systems when considering the versatility of the system used for a variety of procedures which are routinely carried out in a modern dental practice, including a multitude of soft tissue procedures, such as soft tissue surgery, periodontal therapy as well as being an efficient tool for use in implantology, endodontics and tooth whitening. When compared to "classical" dental techniques, the diode laser offers distinctive advantages, such as the ability to cut, coagulate, ablate or vaporize target tissue elements, enabling dry-field surgery through the sealing of small blood vessels (hemostasis), disinfection of the tissue, reduced post-operative edema (through the sealing of small lymphatic vessels) and decreased amount of scarring, contributing to faster and more effective treatment resulting in improved treatment outcome and increased patient comfort and satisfaction.

Acknowledgements

The authors acknowledge SOLA (society of oral laser application) for their valuable documental support.

\section{References}

[1]. Raj Wadhwan : Lasers in dentistry - An introduction to new technology; International Dentistry SA vol. 9 , No. 2

[2]. Coluzzi DJ. Fundamentals of dental lasers:science and instruments. Dent Clin North Am 2004; 48(4):751-770.

[3]. Samo Pirnat: Versatility of an $810 \mathrm{~nm}$ Diode Laser in Dentistry: An Overview: Journal of Laser and Health Academy Vol. 2007 No. $41-8$.

[4]. V. K. Kononenko History and Developments of Semiconductor Lasers :Stepanov Institute of Physics NASB, Independence Ave., 68, 220072 Minsk, Belarus

[5]. Sebeena Mathew, Deepa Natesan Thangaraj: Lasers In Endodontics: JIADS VOL -1Issue 1 Jan- March,2010 ,31

[6]. Academy of laser dentistry.2008; 1-18

[7]. Stubinger S, Saldamli B, Jurgens P, Ghazal G, Zeilhofer HF.Soft tissue surgery with the diode laser- theoretical and clinical aspects. Schweiz Monatsschr Zahnmed. 2006; 116(8): 812-820

[8]. Parker S. Lasers and soft tissue: 'fixed' soft tissue surgery. Br Dent J. 2007 Mar 10; 202(5): $247-253$.

[9]. Bladowski M, Konarska-Choroszucha H, Choroszucha T. Comparison of Treatment Results of Recurrent Aphthous Stomatitis (RAS) with Low- and High-power Laser Irradiation vs a Pharmaceutical Method (5-year Study). J Oral Laser Applications 2004, 3: $191-209$

[10]. Lanning S K, Waldrop T C, Gunsolley J C, Maynard J G. Surgical crown lengthening: evaluation of the Biological width. J Periodontol 2003; 74: 468-474. 
[11]. Gracis S, Fradeani M, Celletti R, Bracchetti G. Biological integration of aesthetic restorations: factors Influencing appearance and long-term success. Periodontol 2000. 2001; 27: 29-44.

[12]. Adams T C, Pang P K. Lasers in aesthetic dentistry. Dent Clin North Am 2004; 48: 833- 860,

[13]. Parker S. Lasers and soft tissue: periodontal therapy. Br Dent J. 2007 Mar 24; 202(6): 309-315.

[14]. Parker S. Surgical laser use in implantology and endodontics. Br Dent J. 2007 Apr 14; 202 (7):377-386.

[15]. Martins MC, Abi-Rached RS, Shibli JA, Araujo MW, Marcantonio E Jr. Experimental peri-implantTissue breakdown around different dental implant surfaces: clinical and radiographic evaluation in dogs. Int J Oral Maxillofac Implants 2004; 19: 839-848.

[16]. Shibli JA, Martins MC, Lotufo RF, Marcantonio E Jr. Microbiologic and radiographic analysis of ligature Induced peri-implantitis with different dental implant surfaces. Int J Oral Maxillofac Implants 2003; 18: 383 -390.

[17]. Mombelli A. Etiology, diagnosis, and treatment considerations in peri-implantitis. Curr Opin Periodontol 1997; 4: 127-136.

[18]. Leonhardt A, Renvert S, Dahlen G. Microbial findings at failing implants. Clin Oral Implants Res 1999; 10: $339-345$.

[19]. Maiorana C, Salina S, Santoro F. Treatment of Periimplantitis with Diode Laser: A Clinical Report. J Oral Laser Applications 2002, 2: 121-127

[20] . Koukichi-Matsumoto: Lasers in Endodontics: DCNA. 2000; Vol 44(4): 889-906

[21]. Nobert Gutknecht: Lasers in endodontics. Journal of laser and health academy. 2008; Vol 4; 1-5,

[22]. Gutknecht N, Franzen R, Meister J, Wanweersch L, Mir M. Temperature evolution on human teeth root surface after diode laser assisted endodontic treatment. Lasers Med Sci. 2005 Sep; 20(2):99-103.

[23]. Schoop U, Kluger W, Moritz A, Nedjelik N, Georgopoulos A, Sperr W. Bactericidal effect of different laser systems in the deep layers of dentin. Lasers Surg Med. 2004; 35(2): 111 - 116.

[24]. Sulieman M, Rees JS, Addy M. Surface and pulp chamber temperature rises during tooth bleaching using a diode laser: a study in vitro. Br Dent J. 2006 Jun 10; 200(11): 631-634;

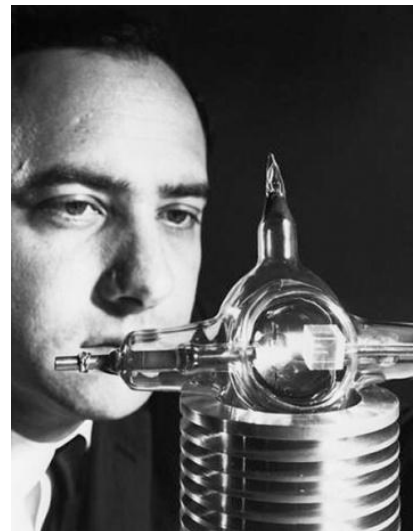

Fig.no.1: Theodore Maiman

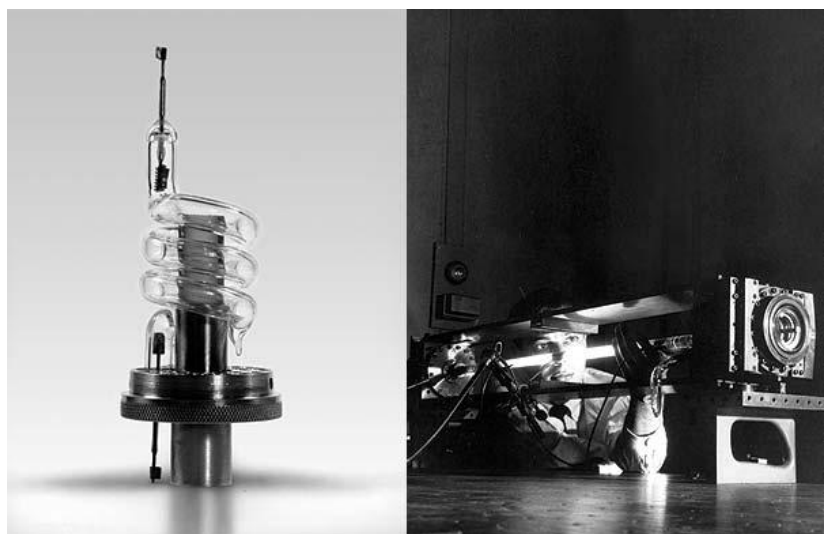

Fig.no.2: Ruby laser

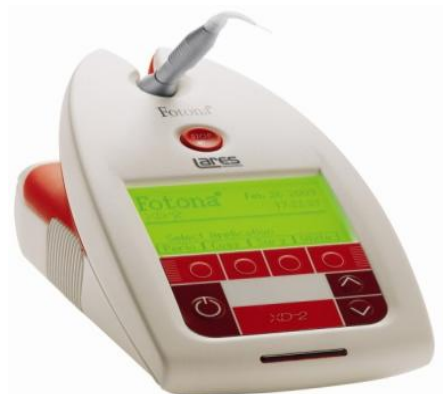

Fig.no.3: Dental Diode laser system (Fotona XD-2)

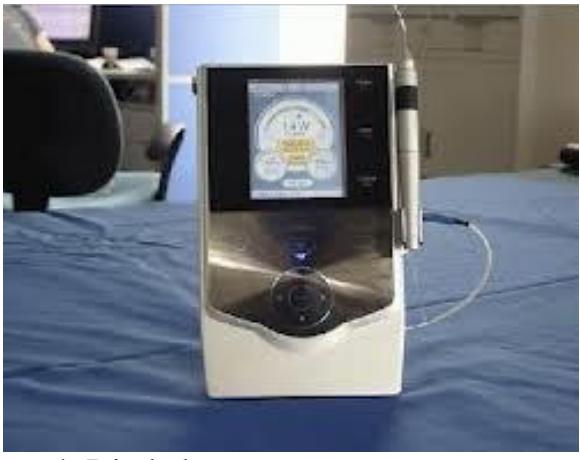

Fig.no.4: Diode laser
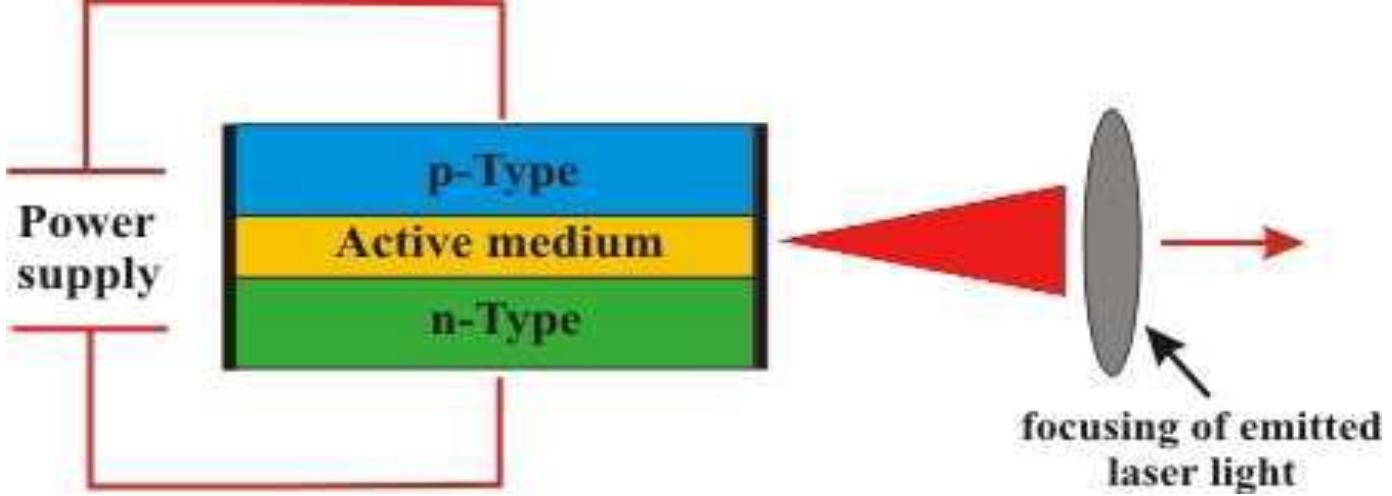

Fig.no.5: Simplified schematic outline of a typical diode laser 